\title{
In vivo measurement of epidermal thickness changes associated with tumor promotion in murine models
}

\section{Kevin G. Phillips}

Oregon Health and Science University

Department of Dermatology

3181 SW Sam Jackson Park Road

L468R Portland, Oregon 97201

\section{Ravikant Samatham \\ Niloy Choudhury \\ James C. Gladish \\ Oregon Health and Science University Department of Biomedical Engineering 3303 SW Bond Avenue \\ Portland, Oregon 97239}

\section{Philippe Thuillier}

Oregon Health and Science University Public Health and Preventative Medicine and the Center for Research on Occupational and Environmental Toxicology

3303 SW Bond Street

Portland, Oregon 97239

\section{Steven L. Jacques}

Oregon Health and Science University

Department of Dermatology

3181 SW Sam Jackson Park Road

L468R Portland, Oregon 97201

and

Oregon Health and Science University

Department of Biomedical Engineering

3303 SW Bond Avenue, Portland, Oregon 97239

\begin{abstract}
The characterization of tissue morphology in murine models of pathogenesis has traditionally been carried out by excision of affected tissues with subsequent immunohistological examination. Excision-based histology provides a limited two-dimensional presentation of tissue morphology at the cost of halting disease progression at a single time point and sacrifice of the animal. We investigate the use of noninvasive reflectance mode confocal scanning laser microscopy (rCSLM) as an alternative tool to biopsy in documenting epidermal hyperplasia in murine models exposed to the tumor promoter 12-O-tetradecanoylphorbol-13-acetate (TPA). An automated technique utilizing average axial rCSLM reflectance profiles is used to extract epidermal thickness values from rCSLM data cubes. In comparisons to epidermal thicknesses determined from hematoxylin and eosin (H\&E) stained tissue sections, we find no significant correlation to rCSLM-derived thickness values. This results from method-specific artifacts: physical alterations of tissue during H\&E preparation in standard histology and specimen-induced abberations in rCSLM imaging. Despite their disagreement, both histology and rCSLM methods reliably measure statistically significant thickness changes in response to TPA exposure. Our results demonstrate that in vivo rCSLM imaging provides epithelial biologists an accurate noninvasive means to monitor cutaneous pathogenesis. @ 2010 Society of Photo-Optical Instrumentation Engineers. [DOI: 10.1117/1.3455508]
\end{abstract}

Keywords: confocal microscopy; cancer; noninvasive imaging; hyperplasia; image segmentation.

Paper 09443SSR received Oct. 2, 2009; revised manuscript received Mar. 15, 2010 ; accepted for publication May 3, 2010; published online Jul. 7, 2010.

\section{Introduction}

Models of skin pathogenesis, e.g., specialized cell culture and mouse models, provide manipulable environments in which cutaneous disease formation in response to wounding or exposure to damaging agents can be investigated systematically. ${ }^{1}$ Molecular techniques, e.g., immunohistochemical staining, Western blot analysis, and quantitative polymerase chain reaction are used to determine the interplay among cells and intracellular signal transduction pathways that result in programmed changes in gene expression and cellular behaviors. These include the regulation of cutaneous cell growth factors influencing differentiation and apoptosis as a result of pathogen exposure. Structural morphology associated with epithelial pathogenesis in mouse models is typically studied through biopsy of affected tissues with subsequent immunohistochemical examination. Ubiquitous tools in epithelial biology research, molecular techniques and excision-

Address all correspondence to Kevin Phillips, Dermatology Research, Oregon Health and Science University, 3181 SW Sam Jackson Park Road-L468R, Portland, Oregon 97210; Tel: 5034948011; E-mail: phillkev@ohsu.edu based histology provide a snapshot of disease progression at the cost of sacrificing the animal and halting the pathological development of interest.

The noninvasive optical detection of tissue changes resulting from pathogenesis using reflectance mode confocal scanning laser microscopy (rCSLM) and optical coherence tomography (OCT) represent novel alternatives to current histological practices in epithelial biology. Both rCSLM and OCT provide three-dimensional images of tissue structures in vivo. Hence, interruption of disease progression through excision of tissues can be circumvented and provides researchers with either an alternative to invasive histology or enables in situ studies of excised tissues to augment current molecular and histological techniques. ${ }^{2}$

As an example of the information afforded by rCSLM, we demonstrate in Figure 1 the similarity of sagittal views of FVB mouse ear skin observed with in vivo rCSLM imaging $(60 \times$ water-coupled objective, NA $=0.9)$ at $488 \mathrm{~nm}$ illumination and standard hematoxylin and eosin (H\&E) staining of a

1083-3668/2010/15(4)/041514/9/\$25.00 @ 2010 SPIE 

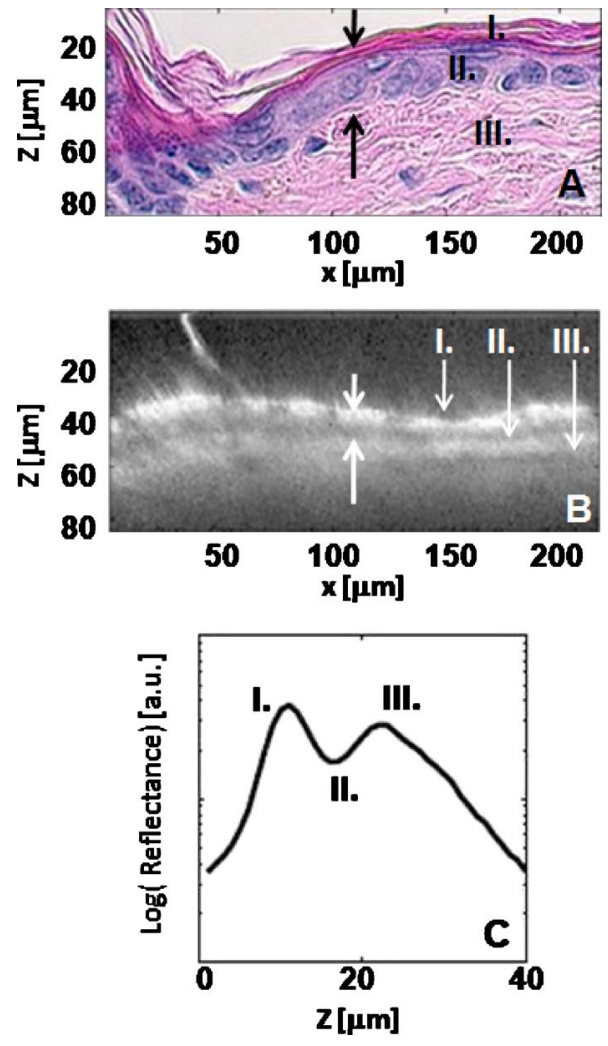

Fig. 1 Comparison of structural features in rCSLM and standard H\&E sections. (a) Standard H\&E section of mouse ear epidermis. (b) Corresponding sagittal view of mouse ear obtained by rCSLM imaging at $488 \mathrm{~nm}$. (c) Axial profile obtained by averaging over a $4 \mu \mathrm{m}$ $\times 4 \mu \mathrm{m} \times 105 \mu \mathrm{m}$ subregion of the confocal image data; see Sec. 3 for a full description. In all figures, I is the entrance peak corresponding to the tissue water interface, II is the valley presumed to correspond to the DEJ, and III is the secondary peak resulting from backscatter by the upper dermis. The epidermis is the region between I and II; the dermis is the region beyond III.

5- $\mu$ m-thick skin section. In Figs. 1(a) and 1(b), the stratum corneum is the topmost layer, denoted by $\mathrm{I}$, in both the $\mathrm{rC}$ SLM image and H\&E section. Below that is the epidermis, the region between I and II, composed of keratinocytes, appearing blue-purple, due to hematoxylin nuclear staining in the H\&E section and as an attenuating brightness that ends in a well-defined dark region in the rCSLM image. The third layer seen in the sagittal images, denoted III, is the beginning of the upper dermis, the red-pink layer, due to eosin staining in the $\mathrm{H} \& \mathrm{E}$ sections, and the second bright layer in the $\mathrm{rC}$ SLM image. rCSLM is able to distinguish these layers owing to the reflectance arising from refractive index mismatch at each of the layer interfaces: for humans, the stratum corneum (hydrated) $n_{s c}=1.51$, viable epidermis $n_{v e d}=1.34$, and dermis $n_{d}=1.4$ (Ref. 3).

Averaged axial reflectance profiles recapitulate the findings in the sagittal images ${ }^{2}$ [see Fig. 1(c)]: an entrance peak as the signal crosses from the coupling medium of the objective lens (here, water) into the stratum corneum, labeled I, in Fig. 1(c), with a second peak, labeled III in Fig. 1(c), occurring at greater depth corresponding to the papillary-reticular dermis junction $(\mathrm{PRJ})^{2}$ or to fibrous structures in the upper dermis. ${ }^{4}$ In comparative studies between OCT and cryostat histology, the local minima (valley), labeled II in Fig. 1(c), between the entrance peak and the secondary peak has been shown to correspond to the dermal-epidermal junction (DEJ). ${ }^{5}$

Using the interpeak and (first) peak-valley distances of averaged axial scans as measures of epidermal thickness, we propose the use of rCSLM to monitor epidermal hyperplasia in FVB mice exposed to the tumor promoting agent 12-Otetradecanoylphorbol-13-acetate (TPA) as a replacement of standard invasive histology for time course studies in the same animal. To validate this method and investigate the dynamic range of the rCSLM-based measurement, we perform rCSLM imaging on mice given acute and chronic doses of TPA. Subsequent to imaging, animals are sacrificed so that rCSLM-derived thickness values can be compared to standard H\&E-determined epidermal thicknesses using bright-field microscopy.

The paper is organized as follows: In Sec. 2 we present our chemical carcinogenesis, rCSLM imaging, and H\&E sectioning protocols. In Sec. 3 the image segmentation algorithm to determine epidermal thickness and corresponding thickness mappings over the field of view is presented. Section 4 contains our rCSLM and standard H\&E results and discussion. We conclude in Sec. 5.

\section{Materials and Methods}

\subsection{Chemical-Induced Cell Proliferation in Mouse Models}

All animal studies were approved by the Oregon Health and Science University Institutional Animal Care and Use Committee. Chemical inducement of cutaneous carcinogenesis in mouse skin is a highly regulable model of cancer formation characterized by a three-step process: tumor initiation, promotion, and progression. ${ }^{6}$ We focus on the reversible effects of tumor promoters: specifically, those of TPA in the absence of tumor initiation. Cell proliferation induced by the tumor promoter TPA has a fast onset and is reproducible in the same animal. ${ }^{1,6}$ Sixteen total FVB mice (Charles River; 10 to 12 weeks old) were used in the acute and chronic arms of the study. The eight mice in the acute arm were given a topical dose of $2.5 \mu \mathrm{g}$ of TPA dissolved in acetone. TPA + acetone was painted on the right ear of the FVB mice, while the left ear was treated only with acetone. At $24 \mathrm{~h}$ after TPA administration, confocal imaging was performed followed by tissue excision and H\&E sectioning of the imaged area. The eight mice in the chronic arm of the study were treated with the same TPA dose as those in the acute arm but were given a daily dose of TPA for five days. Control ears were painted with acetone each day. Imaging was performed on the sixth day.

\section{2 rCSLM Imaging Protocol}

Mouse imaging was performed using rCSLM (see Fig. 2). The rCSLM system consists of an argon ion laser, $\lambda=488 \mathrm{~nm}$, with an average output power of $100 \mathrm{~mW}$, providing illumination through a water-coupled objective lens with numerical aperture (NA) 0.90 and $60 \times$ magnification (Olympus America, Melville, New York). The object lens was set up in an inverted orientation. $(x, y)$ translation of the focus was achieved using a scanning assembly consisting of two galva- 


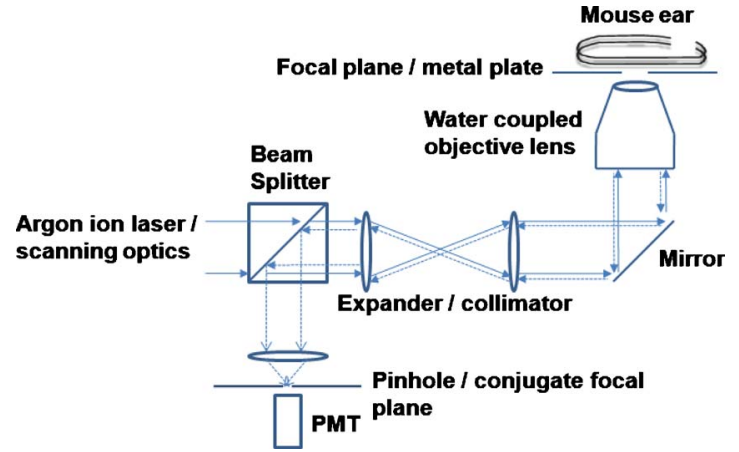

Fig. 2 Ray diagram of the rCSLM system. Illuminating rays are solid; the reflected rays from the sample plane are dashed. The rCSLM system consists of an argon ion laser, $\lambda=488 \mathrm{~nm}$, a water-coupled objective lens with numerical aperture (NA) 0.90 and $60 \times$ magnification. Translation of the focus was achieved using a scanning assembly consisting of two galvanometer mirrors and a pair of relay lenses. Confocal detection of light was achieved by collimating the reflected signal and redirecting the beam toward a lens/pinhole/PMT assembly.

nometer mirrors (Nutfield Technology, Inc., Windham, New Hampshire) and a pair of relay lenses that directed the laser beam into the rear of the objective lens at varying angles. Confocal detection of light originating from the focus in the mouse tissue was achieved by recollimating the reflected signal and then returning the signal through the optical train until the beam was redirected by a beamsplitter toward a lens/ pinhole/photomultiplier tube (PMT) assembly (see Fig. 2).

The $z$ position of the sequential $(x, y)$ scans was incremented in $1-\mu \mathrm{m}$ steps by computer control of the translation stage. The spatial resolution along directions $x$ and $y$ is $0.4 \mu \mathrm{m}$. Data was acquired using an A/D converter controlled by LabView software (National Instruments Corp., Austin, Texas), and image reconstruction was conducted using MATLAB software (The Mathworks, Inc., Natick, Massachusetts).

An FVB mouse was placed in the supine position so that the dorsal side of the ear could be imaged through a warmed metal plate with a 3-mm-diam hole. To restrict movement of the tissue volume under investigation, the ear of the mouse was secured by placing a glass coverslip on the far side of the ear. The reflectance profile from the center of each ear was resolved using confocal detection through the 3-mm hole. The mouse was anesthetized using vaporized isoflurane. The breathing rate of the mouse was regulated by providing $0.2 \mathrm{l} / \mathrm{min}$ oxygen and $0.8 \mathrm{l} / \mathrm{min}$ air mixed with the isoflurane vapor $(1.5 \% \mathrm{~V} / \mathrm{V}$ at $1 \mathrm{ATM})$.

\subsection{Histology Protocol}

Black fiduciary marks on the mouse ears were used to demarcate rCSLM imaged sites. Tissue from these sites was then excised and fixed in formalin for $24 \mathrm{~h}$. The tissue samples were then embedded in paraffin wax, and tissue from the central region of the fiduciary mark was cut into 5-micron-thick sections and plated five sections to a slide. The plated sections were then stained in hematoxylin and eosin.

Image acquisition of $H \& E$ stained tissue sections was performed on a Leica microscope at $60 \times$ (Leica Microsystems, Bannockburn, Illinois) with a CCD camera. Images were loaded in JPG format into Image-Pro Plus (Leeds Precision
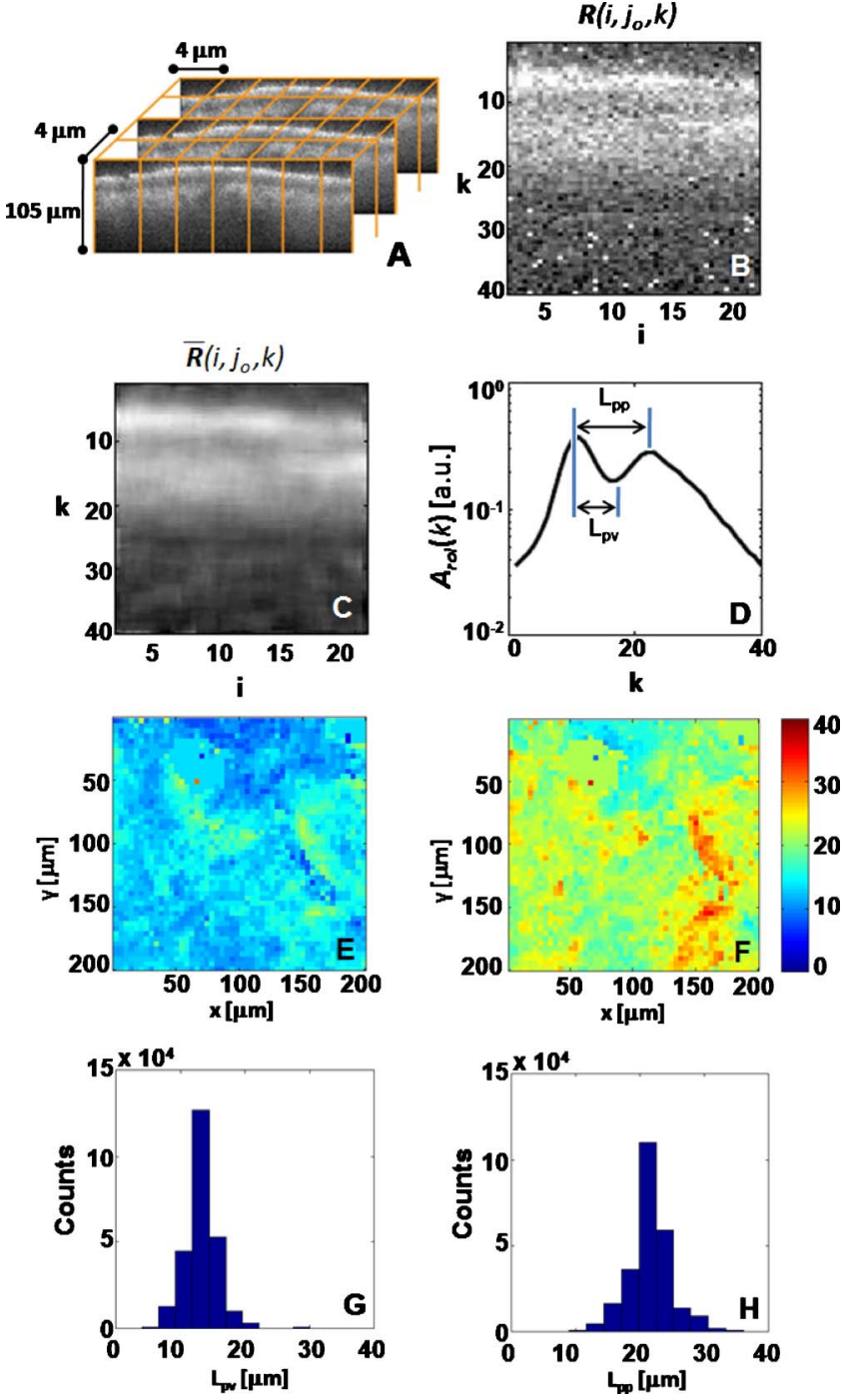

Fig. 3 Automated image processing and thickness mapping. (a) Division of $\boldsymbol{R}(i, j, k)$ into $4 \mu \mathrm{m} \times 4 \mu \mathrm{m} \times 105 \mu \mathrm{m}$ ROI. (b) Sagittal view at fixed $j=j_{o}, \boldsymbol{R}\left(i, j_{o}, k\right)$, raw data. (c) Sagittal view of ROI, after moving average smoothing along the $i$ direction. (d) Average axial reflectance profile, $A_{r o i}(k)$, obtained by averaging for all $i, j$ in the ROI. $L_{p v}$ is the peak-to-valley minima distance along $z . L_{p p}$ is the peak-to-peak distance along $z$. The thickness values for each ROI are then plotted as thickness maps: (e) $L_{p v}$ thickness map (2500 ROI). (f) $L_{p p}$ thickness map. The color bar units are $\mu \mathrm{m}$ and are common to both thickness maps. (g) Histogram of $L_{p v}$ thickness. (h) Histogram of $L_{p p}$ thickness. (Color online only.)

Instruments, Inc., Minneapolis), where the thickness of the epidermis was determined by hand-drawn line segments connecting the intact stratum corneum to the dermal-epidermal junction. The Image-Pro Plus software recorded the length of each line segment drawn. Twenty line segments were drawn on each of the five sections per slide for a total population of 100 thickness measurements made per ear. The mean and standard deviation of this population of thickness values was used as a second means of determining the response of mouse epidermis to the tumor promoter TPA.

\section{3 rCSLM Image Segmentation}

The reflectance from the rCSLM system is stored in the threedimensional matrix $\boldsymbol{R}_{i j k}$. For notational convenience, we write 

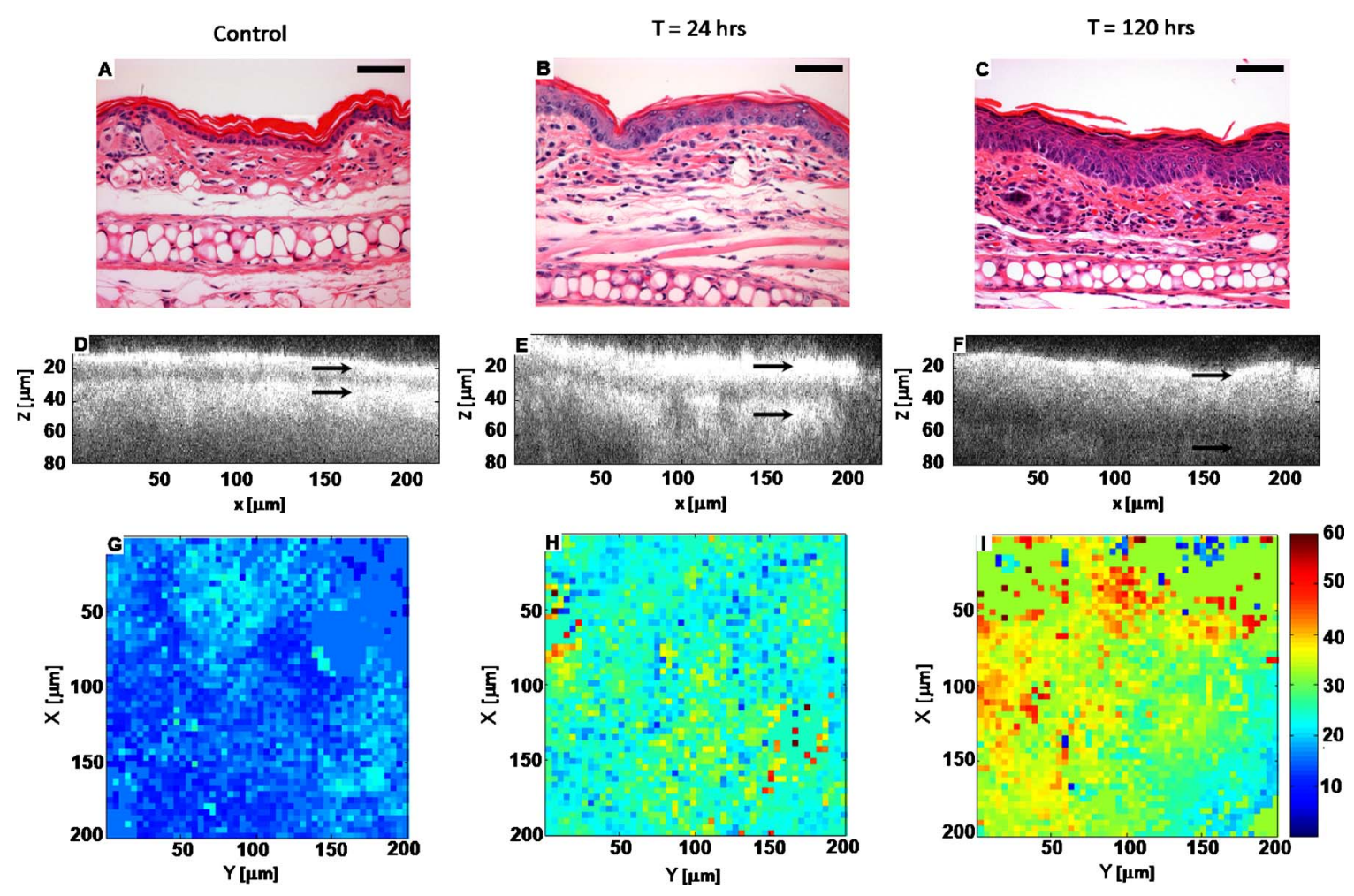

Fig. 4 Comparison of morphological features in untreated, acute, and chronically exposed mouse ear skin. The column of images in the figure correspond to control ears (column 1), acutely treated ears (column 2), and chronically treated ears (column 3). The top row [(a) to (c)] is a standard H\&E stain of 5- $\mu$ m-thick sections embedded in paraffin wax. The middle row images [(d) to (f)] correspond to sagittal views of the confocal scan with brightness values presented on a logarithmic scale in arbitrary units. Panels (g) to (i) are example $L_{p p}$ thickness maps with 2500 ROls (see Sec. 3 for a description) for each treatment type. The thickness maps are plotted with the common color bar at right with units of $\mu \mathrm{m}$. (Color online only.)

$\boldsymbol{R}(i, j, k)$. The indices $i, j$ correspond to pixel values of the PMT recorded at each increment, $k$, of the $z$-translation stage. In the system described, the indices range according to 1 $\leqslant i, j \leqslant N=483$ and $1 \leqslant k \leqslant 100$. The true spatial location of a pixel value corresponding to transverse locations $(x, y)$ is the product of the pixel values $(i, j)$ and the resolution along each spatial direction, given by $\mathrm{d} x=\mathrm{d} y=0.4 \mu \mathrm{m}-\mathrm{e}$.g., $x=i \times \mathrm{d} x$. Spatial locations along the $z$ direction are determined similarly, although with an additional correction factor accounting for refractive index mismatch between the water-coupling lens $\left(n_{0}=1.33\right)$ and the mouse ear tissue $(n=1.4){ }^{2}$ $z=\left(n / n_{o}\right) k \mathrm{~d} z$. Hence, the total volume interrogated by the confocal system is of size $200 \mu \mathrm{m} \times 200 \mu \mathrm{m} \times 105 \mu \mathrm{m}$.

The numerical determination of the epidermal thickness from confocal images is carried out using an automated algorithm that is comprised of the following steps (see Fig. 3 as a visual reference):

1. $\boldsymbol{R}(i, j, k)$ is first divided into 2500 local regions of interest (ROIs) $4 \mu \mathrm{m} \times 4 \mu \mathrm{m} \times 105 \mu \mathrm{m}$ in size (or 10 pixel $\times 10$ pixel $\times 100$ pixel); see Fig. $3($ a).

2. For each $i, j, k$ in the ROI, the reflectance is smoothed through a moving average procedure along the $i$ direction:

$$
\overline{\boldsymbol{R}}(i, j, k)=\frac{1}{3}[\boldsymbol{R}(i-1, j, k)+\boldsymbol{R}(i, j, k)+\boldsymbol{R}(i+1, j, k)] .
$$

For a fixed value of $j=j_{o}$, we present $\boldsymbol{R}\left(i, j_{o}, k\right)$ (raw) and $\overline{\boldsymbol{R}}\left(i, j_{o}, k\right)$ (smoothed), in Figs. 3(b) and Figure 3(c), respec- tively. This averaging procedure helps eliminate speckle noise in the raw confocal data while preserving the reflectance from the distinct layers of the mouse epidermis.

3. An average axial profile, $A_{\text {roi }}(z)$, is obtained for each ROI by averaging the local axial reflectance profiles for each $(i, j)$ location in the ROI,

$$
A_{r o i}(k)=\frac{1}{N^{2}} \sum_{i, j \in R O I} \overline{\boldsymbol{R}}(i, j, k) .
$$

The pixel value dependence, $k$, is then scaled to obtain the average axial reflectance as a function of the depth $z$ :

$$
A_{\text {roi }}(z)=A_{\text {roi }}\left[\left(n / n_{o}\right) k \mathrm{~d} z\right],
$$

as shown in Fig. 3(d).

4. The thickness of the epidermis is then monitored by two means: the peak-to-valley minima distance, $L_{p v}$, in the average A-scan for each ROI, and the peak-to-peak distance, $L_{p p}$; see Fig. 3(d). The inflection points of the A-scan are determined using peakdet, a MATLAB peak detection algorithm that looks for a high point surrounded by points lower by a number $\alpha$ on both sides. ${ }^{7}$

5. $L_{p v}$ and $L_{p p}$ determined for each ROI are plotted in a thickness map; see Figs. 3(e) and 3(f). The color bar denotes thicknesses in microns and is common to both thickness maps. 


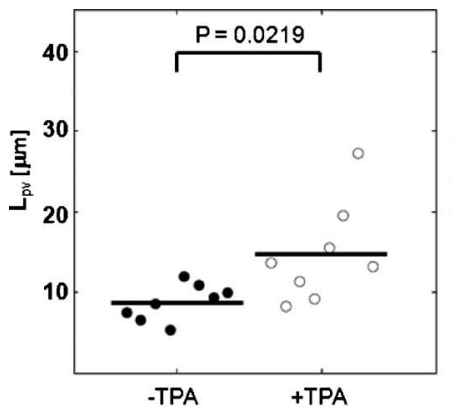

(a)

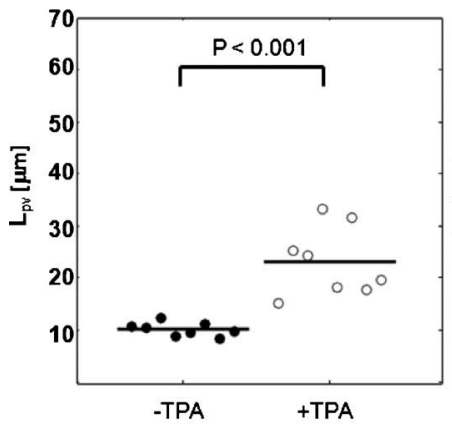

(d)

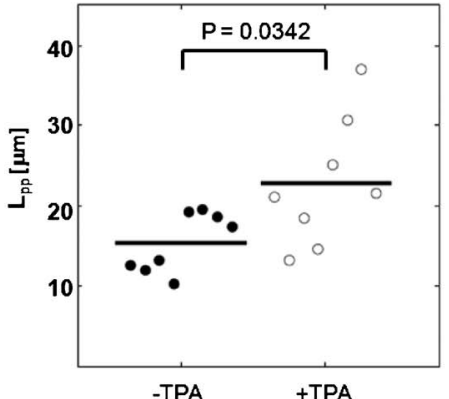

(b)

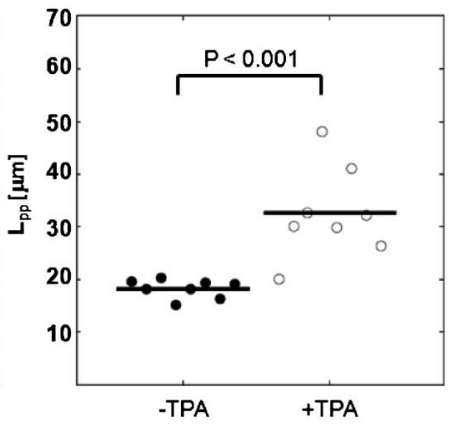

(e)

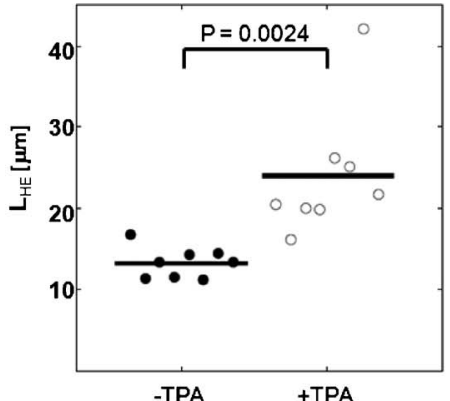

(c)

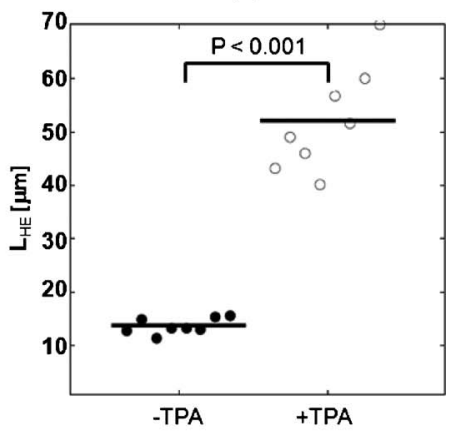

(f)

Fig. 5 Epidermal thickness in untreated (-TPA) and treated (+TPA) mouse ear skin: acute and chronic exposures. Panels (a), (b), and (c) correspond to mice treated with a single dose of TPA. Panels (d), (e), and ( $f$ ) correspond to mice treated with 5 doses of TPA. (a) and (d) present changes in $L_{p v^{\prime}}$ (b) and (e) present changes in $L_{p p^{\prime}}$ (c) and (f) are H\&E-derived thickness.

6. The average value and standard deviation of the population of thickness values in each map gauges the bulk tissue response in the full tissue volume under interrogation; see Figs. 3(g) and 3(h).

\section{Results}

\subsection{Epidermal Response to TPA}

In Figure 4, we demonstrate the degree of epidermal proliferation in response to acute and chronic exposure to TPA with sagittal standard H\&E sections and sagittal rCSLM images. The columns of images in the figure correspond to control ears (column 1), acutely treated ears (column 2), and chronically treated ears (column 3). Epidermal proliferation is evident in the the H\&E sections as an increase in the amount of nucleated cells (stained purple) present in the epidermis; see panels (a) to (c). A similar change is seen in the rCSLM images as the two bright layers (the top one corresponding to the stratum corneum, the bottom to the upper dermis) become increasingly separated in response to the amount of TPA exposure; see panels (d) to (f). The reflectance from the upper dermis in the chronic sagittal image becomes reduced owing to the increasing amount of cells present between the stratum corneum and the upper dermis. These cells act to scatter and degrade the confocal signal. The final row of images, panels (g) to (i) in Fig. 4, are example $L_{p p}$ thickness maps (see Sec. 3 for a description), for each treatment type. The thickness maps are plotted with the common color bar at right with units of $\mu \mathrm{m}$.

In Figure 5, we present a comparison of epidermal thickness between control and treated ears in the acute study, panels (a) to (c), and the chronic study, panels (d) to (f). Panels (a) and (d) are $L_{p v}$ comparisons, (b) and (e) are $L_{p p}$ comparisons, and (c) and (f) are $L_{H E}$ comparisons. In every case, a statistically significant thickness change in epidermal thickness is observed.

Figure 6 presents the fold changes (denoted FC and defined as the ratio of the thickness of treated ears to control ears) in epidermal thickness associated with acute and chronic TPA exposure for each epidermal thickness measurement type. On average, we find that PV fold changes are greater than PP fold changes in both the acute and chronic treatment regimens. Average PV thicknesses correspond better with the magnitude of fold changes observed in H\&E. This makes sense, as $L_{p p}$ is thought to measure the distance between the stratum corneum and the upper dermis. ${ }^{2}$

Complete $L_{p v}, L_{p p}$, and $L_{H E}$ thicknesses along with their standard deviations, and for rCSLM-derived values, the number of ROIs used in the image sectioning algorithm are reported in Table 1 (acute study) and Table 2 (chronic study) in the appendix. On average, we find that in the acute study $L_{p v}$ for control skin is $8.7 \mu \mathrm{m}$ and that of once-treated TPA skin is $14.7 \mu \mathrm{m}(P=0.0219)$, with an average fold change of 1.69 . Correspondingly for $L_{p p}$ thickness values, we observe a thickness of $15.4 \mu \mathrm{m}$ in control tissue and a thickness of $22.7 \mu \mathrm{m}$ in once-treated TPA skin $(P=0.0342)$, with an average fold change of 1.46. Standard H\&E showed that untreated skin was $13.3 \mu \mathrm{m}$ thick, while once-treated TPA skin was $24 \mu \mathrm{m}$ thick $(P=0.0024)$, with an average fold change of 1.81 .

In the chronic study, we observed that $L_{p v}=10.2 \mu \mathrm{m}$ for control skin, $23.2 \mu \mathrm{m}$ for 5 times treated TPA skin $(P<0.001)$, with an average fold change of 2.3. $L_{p p}$ thicknesses were $18.2 \mu \mathrm{m}$ for control, $32.5 \mu \mathrm{m}$ for treated 
Phillips et al.: In vivo measurement of epidermal thickness changes associated with tumor promotion...

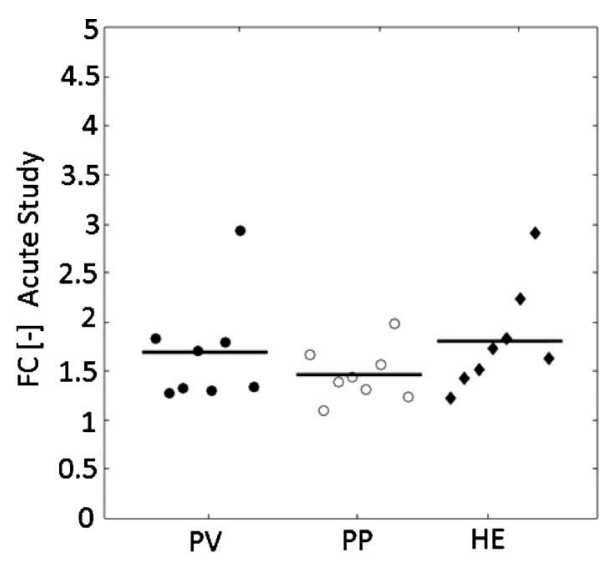

(a)

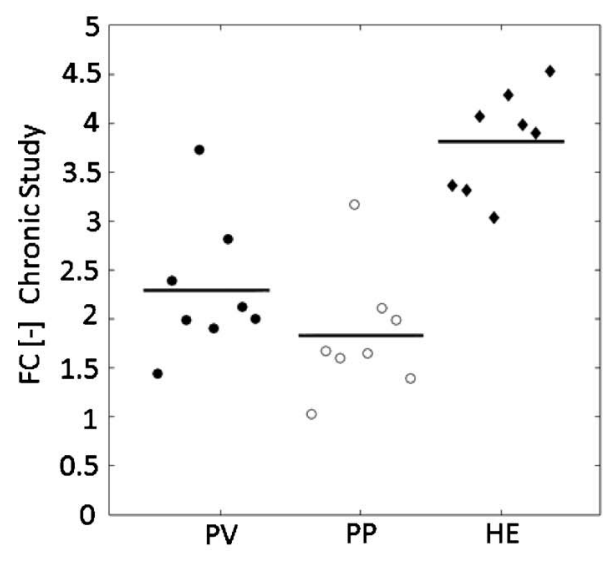

(b)

Fig. 6 Fold changes in H\&E and rCSLM epidermal thickness values in response to TPA. Fold change is defined as the ratio of the thickness value in the presence of TPA to the thickness value of the control: (a) acute study, (b) chronic study.

$(P<0.001)$, with an average fold change of 1.82. Standard H\&E showed $13.7 \mu \mathrm{m}$ for control, $52.1 \mu \mathrm{m}$ for treated $(P<0.001)$, with an average fold change of 3.8 .

\subsection{Comparison of rCSLM and H\&E Methods}

To quantify the agreement between rCSLM and H\&E thickness measurement methods, we plot the measured thicknesses from each method against one another and look for a linear correlation; see Fig. 7. The square of the linear correlation value is presented and denoted $r_{p v}^{2}\left(r_{p p}^{2}\right)$ when comparing peak-to-valley (peak-to-peak) determined thicknesses and $\mathrm{H} \& \mathrm{E}$ determined thicknesses.

Figure 7(a) presents a scatter plot of epidermal thickness for control data for the acute study for mouse ears exposed only to acetone; $r_{p v}^{2}=0.03, r_{p p}^{2}=0.01$. Similar results were obtained for the chronic controls (data not shown). In Fig. 7(b), we present a comparison of epidermal thickness values determined for mice in the acute arm of the study: $r_{p v}^{2}=0.90, r_{p p}^{2}$ $=0.82$. Panel $(\mathrm{c})$ presents thickness values for mice in the chronic arm of the study: $r_{p v}^{2}=0.18, r_{p p}^{2}=0.10$.

We also investigate the possibility of a linear correlation among the fold changes in thickness measured by rCSLM and H\&E. In Fig. 8, we plot the fold changes against each other and observe in panel (a) that fold changes for mice given a single dose of TPA: $r_{p v}^{2}=0.62, r_{p p}^{2}=0.45$; and in panel (b) that fold changes for mice given five doses of TPA: $r_{p v}^{2}=0.21$, $r_{p p}^{2}=0.18$.

\section{Discussion}

In both the acute and chronic treatments where weak and strong tissue responses are observed, rCSLM measures statistically significant tissue alterations; see Fig. 8. We posit that the general lack of linear correlation between rCSLM imaging and standard $\mathrm{H} \& \mathrm{E}$ regarding both the magnitude of epidermal thickness and fold changes incurred during TPA-induced hyperplasia arises from artifacts encountered when using either method.

\subsection{H\&E Artifacts}

In $H \& E$ preparations, tissues are formalin fixed and then cut into thin sections before staining. This process shrinks the dermal portions of the tissue as it removes most of the water content. It is also possible that the tissue may expand along the axial $z$ direction and shrink along the transverse direction once the lateral forces of surrounding tissue are removed. Last, the stratum corneum is devastated by the sectioning process. Typically, it can be seen flaking off the tissue section.

\section{2 $\operatorname{rCSLM}$ Artifacts}

Variations in the refractive index act to shift the focal plane of the objective lens depending on the refractive index mismatch at layer interfaces. Using a geometrical analysis of marginal rays from the objective (see appendix $\mathrm{C}$ of Ref. 8, for example), it is evident that the focus can be shifted inward (when $\left.n_{2}>n_{1}\right)$ or outward $\left(n_{2}<n_{1}\right)$ with respect to the position of the focus, in the case of index matched layers. This is a layer-dependent process. In the present analysis, we have assumed only an average contribution from this effect for all layers: we have scaled the known objective position by $n / n_{o}$; see Sec. 3. This accounts for a $5 \%$ shift in the axial location of the focus, which is not enough to bring about agreement with H\&E-determined thicknesses.

A second artifact of confocal imaging arises from image segmentation. In Table 1, we present the percentage of the possible 2500 ROIs used in the determination of $L_{p v}$ and $L_{p p}$, denoted $N(\%)$. On average, we excluded $28.3 \%$ of ROIs in the acute study and $33.2 \%$ in the chronic study because they do not exhibit the double peak feature illustrated in Fig. 3(d). Gambichler and researchers ${ }^{5}$ have reported a similar percentage $(40 \%)$ of unusable average axial profiles in their work on documenting epidermal thickness in humans using OCT. In the thickness maps presented, those regions for which a thickness could not be determined are set to the population average. For H\&E thickness measurements, the total number of measurements is always 100 . The inability of certain ROIs to provide epidermal thickness information is due to the presence of inhibiting anatomical features, most notably hair follicles and sebaceous glands, that disrupt the regular epidermal-dermal structure. For mice chronically exposed to TPA, the second peak corresponding to the upper dermis was more difficult to find. This in turn produced a greater standard deviation for the reported thicknesses; see Table 2 of the appendix. The upper dermis signal degrades, as light is attenu- 
Table 1 Acute study: FVB mouse ear epidermal thickness values in the absence of TPA and $24 \mathrm{~h}$ post $2.5 \mu \mathrm{g}$ topical application of TPA. $N(\%)$ is the percentage of the 2500 ROls used to determine epidermal thicknesses. $L_{p v} L_{p p}$, and $L_{H E}$ are the average peak-to-valley, peak-to-peak, and H\&E-based epidermal thicknesses. $\sigma_{p v,} \sigma_{p p}$ and $\sigma_{H E}$ are the standard deviations.

\begin{tabular}{|c|c|c|c|c|c|c|c|}
\hline Mouse-TPA & $N(\%)$ & $L_{p v}[\mu \mathrm{m}]$ & $\sigma_{p v}[\mu \mathrm{m}]$ & $L_{p p}[\mu \mathrm{m}]$ & $\sigma_{p v}[\mu \mathrm{m}]$ & $L_{H E}[\mu \mathrm{m}]$ & $\sigma_{p v}[\mu \mathrm{m}]$ \\
\hline 1 & 85.76 & 7.8 & 2.7 & 13.3 & 3.8 & 16.8 & 3.5 \\
\hline 2 & 76.32 & 6.9 & 1.8 & 12.7 & 2.7 & 11.4 & 2.7 \\
\hline 3 & 80.68 & 9.0 & 2.8 & 14.0 & 3.6 & 13.3 & 1.9 \\
\hline 4 & 76.92 & 5.6 & 2.4 & 10.8 & 4.1 & 11.5 & 2.3 \\
\hline 5 & 67.44 & 11.9 & 2.6 & 19.2 & 3.2 & 14.3 & 2.6 \\
\hline 6 & 63.36 & 10.9 & 2.9 & 19.5 & 5.1 & 11.2 & 1.6 \\
\hline 7 & 94.48 & 9.2 & 3.6 & 18.7 & 4.8 & 14.5 & 4.7 \\
\hline 8 & 46.12 & 9.9 & 3.8 & 17.5 & 5.3 & 13.4 & 1.8 \\
\hline \multicolumn{8}{|l|}{ Mouse + TPA } \\
\hline 1 & 55.96 & 14.4 & 3.6 & 21.1 & 5.2 & 20.5 & 3.1 \\
\hline 2 & 50.16 & 8.7 & 2.1 & 13.2 & 3.1 & 16.2 & 2.3 \\
\hline 3 & 75.64 & 11.9 & 3.2 & 18.5 & 4.3 & 20.1 & 4.9 \\
\hline 4 & 80.88 & 9.6 & 4.3 & 14.6 & 5.3 & 19.9 & 3.4 \\
\hline 5 & 94.32 & 15.5 & 5.0 & 26.2 & 5.0 & 26.2 & 3.8 \\
\hline 6 & 82.48 & 19.6 & 4.4 & 25.1 & 4.6 & 25.1 & 4.8 \\
\hline 7 & 83.96 & 27.2 & 4.6 & 42.1 & 4.7 & 42.1 & 9.7 \\
\hline 8 & 94.16 & 13.2 & 2.5 & 21.7 & 3.3 & 21.7 & 7.5 \\
\hline
\end{tabular}

ated more by the increased number of cells that the signal has to travel through in tissues experiencing hyperplasia. This is evident in the structural image; see Fig. 4(f).

Last, we mention that the loading of the tissue by a glass coverslip and metal weight $(5 \mathrm{~g})$ may also induce a flattening effect of the tissue during rCSLM imaging. We imaged in the presence and absence of the glass coverslip/weight combination and found that the compaction of the ear had no effect on changing the measured epidermal thickness.

\section{Conclusion}

In this study, we have demonstrated the ability of rCSLM imaging to provide a robust measurement of epidermal thickness change. Our results indicate that the peak-to-valley distances in averaged axial scans are more sensitive to epidermal changes than the peak-to-peak distances. The co-registration of absolute thickness values reported by the different measurement methods remains elusive: in general, the values of $L_{H E}$ are greater than $L_{p v}$ and $L_{p p}$, with no linear correlation among the rCSLM and H\&E derived values. Efforts to reconcile the two measurements by taking account of specimeninduced abberations and potential compaction of tissue by restraining devices during imaging do not account for enough of a thickness change to bring about agreement. We conjecture that the greater magnitude of the $L_{H E}$ values in comparison to the $L_{p v}$-based thicknesses is attributed to the stretching of the tissue sections in the $z$ direction post-excision. This possible stretching in no way hampers the ability of $H \& E$ to remain a gold standard in documenting disease progression. It merely hampers the ability for correlation of $\mathrm{H} \& \mathrm{E}$ with axial features of rCSLM images.

Future studies of hyperplasia in response to tumor promoters should be accompanied by OCT measurements. OCT is capable of analyzing changes in the dermis. During the course of TPA treatments, we found that the ears increased in total thickness. OCT would be capable of documenting tissue swelling and quantifying the leukocyte infiltration. Our group has taken preliminary steps in this direction. ${ }^{9}$

\section{Acknowledgments}

We thank the reviewers for their very helpful and constructive criticisms of the original draft. Special thanks go to Julia Sonka, Justine Brown, Amanda Hillman, Sawan Hurst, James Raymond, Aaron Wortham, and Trevor Levin for technical assistance. Histology was performed by Carolyn Gendron of the Chris Corless Lab. We thank Dr. Jayme Gallegos, Dr. 
Phillips et al.: In vivo measurement of epidermal thickness changes associated with tumor promotion...

Table 2 Chronic study: FVB mouse ear epidermal thickness values in the absence of TPA and $24 \mathrm{~h}$ post 5-day daily treatment with $2.5 \mu \mathrm{g}$ topical application of TPA. $N(\%)$ is the percentage of the $2500 \mathrm{ROIs}$ used to determine epidermal thicknesses. $L_{p v} L_{p p}$, and $L_{H E}$ are the average peak-to-valley, peak-to-peak, and $\mathrm{H} \& \mathrm{E}$-based epidermal thicknesses. $\sigma_{p v}, \sigma_{p p}$, and $\Gamma_{H E}$ are the standard deviations.

\begin{tabular}{|c|c|c|c|c|c|c|c|}
\hline Mouse-TPA & $N(\%)$ & $L_{p v}[\mu \mathrm{m}]$ & $\sigma_{p v}[\mu \mathrm{m}]$ & $L_{p p}[\mu \mathrm{m}]$ & $\sigma_{p v}[\mu \mathrm{m}]$ & $L_{H E}[\mu \mathrm{m}]$ & $\sigma_{p v}[\mu \mathrm{m}]$ \\
\hline 1 & 72.44 & 10.6 & 3.1 & 19.5 & 4.0 & 12.8 & 2 \\
\hline 2 & 55.60 & 10.6 & 6.8 & 18.1 & 7.1 & 14.8 & 2.4 \\
\hline 3 & 68.80 & 12.2 & 3.9 & 20.2 & 4.7 & 11.3 & 1.4 \\
\hline 4 & 60.24 & 8.9 & 5.4 & 15.2 & 5.4 & 13.3 & 2.1 \\
\hline 5 & 63.36 & 9.6 & 2.5 & 18.1 & 3.9 & 13.2 & 2.5 \\
\hline 6 & 96.72 & 11.2 & 2.5 & 19.4 & 3.4 & 13.0 & 1.7 \\
\hline 7 & 87.20 & 8.3 & 1.7 & 16.2 & 2.6 & 15.4 & 2.7 \\
\hline 8 & 93.24 & 9.8 & 3.8 & 19.0 & 4.6 & 15.5 & 3.0 \\
\hline \multicolumn{8}{|l|}{ Mouse + TPA } \\
\hline 1 & 46.64 & 15.2 & 4.5 & 20.0 & 4.9 & 43.1 & 11.6 \\
\hline 2 & 76.36 & 25.2 & 11.0 & 30.2 & 11.0 & 49.1 & 11.9 \\
\hline 3 & 78.0 & 24.3 & 6.6 & 32.6 & 7.0 & 46.1 & 8.8 \\
\hline 4 & 39.64 & 33.2 & 20.0 & 48.1 & 12.9 & 40.2 & 6.5 \\
\hline 5 & 72.32 & 18.3 & 8.5 & 29.8 & 5.1 & 56.8 & 16.9 \\
\hline 6 & 48.92 & 31.7 & 5.6 & 41.0 & 6.7 & 51.7 & 14.7 \\
\hline 7 & 46.0 & 17.7 & 7.6 & 32.2 & 4.8 & 60.0 & 8.32 \\
\hline 8 & 53.76 & 19.6 & 6.5 & 26.4 & 6.2 & 70.2 & 8.9 \\
\hline
\end{tabular}

Molly Kulesz-Martin, Dr. Wangcun Jia, Dr. Sean J. Kirkpatrick, and Dr. Scott Prahl for useful discussions. Kevin G. Phillips was supported by the National Institutes of Health under the Ruth L. Kirschstein National Research Service Award 5-T32-CA106195-05, "Training in the Molecular Basis of Skin Pathobiology," from the National Cancer Institute and the OHSU Cancer Institute, funded by the National Cancer Institute, CA-069533. It was the great pleasure of the authors to present preliminary data from this study at the Biophotonics 2009 Summer School in Hven, Sweden. We thank Professors Peter E. Andersen and Stefan Andersson-Engels for their

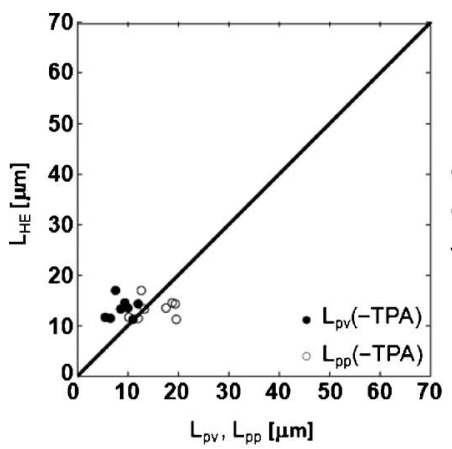

(a)

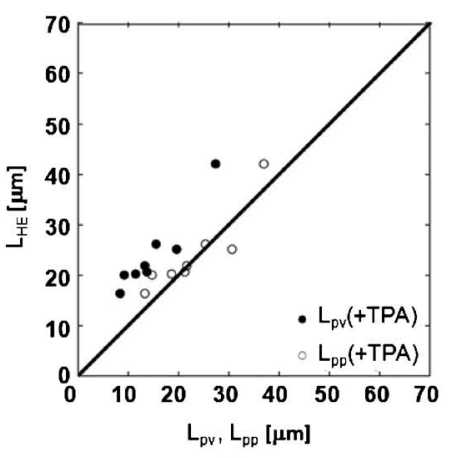

(b)

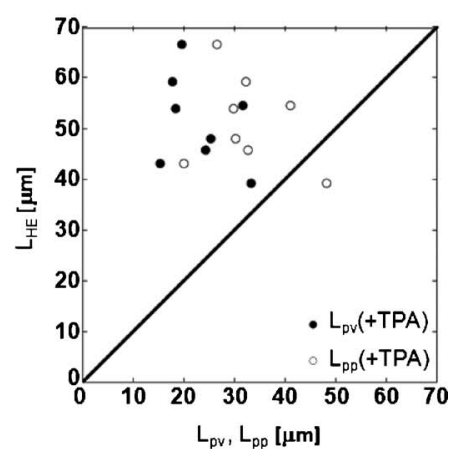

(c)

Fig. 7 Comparison of H\&E and rCSLM epidermal thickness values. (a) Control data for mouse ears exposed only to acetone; $r_{p v}^{2}=0.03, r_{p p}^{2}=0.01$. (b) Comparison of epidermal thickness values determined for mice given a single dose of TPA; $r_{p v}^{2}=0.90, r_{p p}^{2}=0.82$. (c) Thickness values for mice given 5 doses of TPA; $r_{p v}^{2}=0.18 ; r_{p p}^{2}=0.10$. 


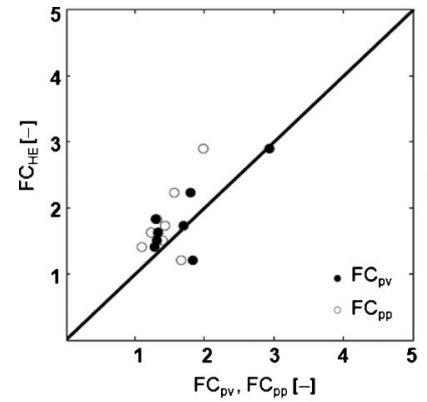

(a)

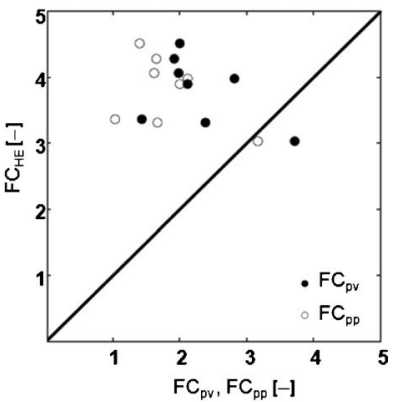

(b)
Fig. 8 Comparison of H\&E and rCSLM determined epidermal thickness fold changes in response to TPA. (a) Fold changes for mice given a single dose of TPA; $r_{p v}^{2}=0.62, r_{p p}^{2}=0.45$. (b) Fold changes for mice given 5 doses of TPA; $r_{p v}^{2}=0.21, r_{p p}^{2}=0.18$.

ongoing contributions to the optics community-not only in their work but also as organizers of the summer school.

\section{Appendix}

We list the complete tabulated data set obtained in the acute (Table 1) and chronic (Table 2) experiments. $N(\%)$ is the number of viable ROIs used to determine the average epidermal thicknesses $L_{p v}$ and $L_{p p}$ along with their standard deviations $\sigma_{p v}, \sigma_{p p}$. H\&E-based data is also tabulated. In each case, 100 measurements were made. See Sec. 2 for full details.

\section{References}

1. M. Kulesz-Martin, "Biological aspects of multistage carcinogenesis as studied in experimental animals and in cell culture models," in Comprehensive Toxicology, vol. 12, G. T. Bowden and S. M. Fischer, Eds., pp. 7-30, Pergamon, New York (1997).

2. S. Neerken, G. W. Lucassen, M. A. Bisschop, E. Lenderink, and T. Nuijs, "Characterization of age-related effects in human skin: a comparative study that applies confocal laser scanning microscopy and optical coherence tomography," J. Biomed. Opt. 9(2), 274-281 (2004).

3. G. Tearney, M. Brezinski, J. Southern, B. Bouma, M. Hee, and J. Fujimoto, "Determination of the refractive index of highly scattering human tissue by optical coherence tomography," Opt. Lett. 20, 22582260 (1995)

4. T. Gambichler, S. Boms, M. Stucker, A. Kreuter, G. Moussa, M. Sand, P. Altmeyer, and K. Hoffmann, "Epidermal thickness assessed by optical coherence tomography and routine histology: preliminary results of method comparison," J. Eur. Acad. Dermatol. Venereol 20, 791-795 (2006).

5. T. Gambichler, G. Moussa, P. Regeniter, C. Kasseck, M. Hoffmann, F. Bechara, M. Sand, P. Altmeyer, and K. Hoffmann, "Validation of optical coherence tomography in vivo using cyrostat histology," Phys. Med. Biol. 52, N75-N85 (2007).

6. R. Boutwell and A. Sivak, "The function and mechanism of promoters of carcinogenesis," Crit. Rev. Toxicol. 2(4), 419-444 (1973).

7. Available at http://billauer.co.il/peakdet.html.

8. M. Rajadhyaksha, S. Gonzalez, J. M. Zavislan, R. R. Anderson, and $\mathrm{R}$. H. Webb, "In vivo confocal scanning laser microscopy of human skin II: advances in instrumentation and comparison with histology," J. Invest. Dermatol. 113, 293-303 (1999).

9. K. G. Phillips, N. Choudhury, R. Samatham, H. S. Gill, and S. L. Jacques, "Micro-tattoo guided OCT imaging of site specific inflammation," in Biomedical Applications of Light Scattering IV, A. Wax and V. Beckman, Eds., Proc. SPIE 7573, 75730S (2010). 